\title{
Cognitive Radio MIMO Gaussian Broadcast Channels with the Power Constraint
}

\author{
Hai-Lin Xiao · Shan Ouyang • Cheng-Xiang Wang
}

Published online: 18 December 2011

(C) Springer Science+Business Media, LLC. 2011

\begin{abstract}
The cognitive radio multiple-input multiple-output Gaussian broadcast channels are studied where multiple antennas are available for both primary users and secondary users in a spectrum sharing environment, and the sum-rate capacity is also obtained under both the SUs' transmit power constraint and interference power constraint at the primary receivers. The paper principally consists of two steps. First, a duality technique and dirty paper coding are adopted to simplify the channels. Second, we propose an iterative power allocation algorithm to obtain the maximum sum-rate capacity and examine the effects of the constraint parameters on the concerned quantities. Finally, numerical simulation results are presented to validate the proposed theoretical analysis.
\end{abstract}

Keywords Multiple-input multiple-output (MIMO) · Cognitive radio (CR) . Broadcast channels (BCs) · Power allocation

\section{Introduction}

In recent years, the study of cognitive radio (CR) from an information theoretic perspective has gained prominence $[1,2]$. CR allows a secondary user (SU) to opportunistically or concurrently access the spectrum initially allocated to the primary user (PU) provided that certain prescribed constraints are satisfied. Such spectrum access policies require that the transmission power of the secondary transmitter be controlled so as to emit limited interference to the primary receiver [3]. Multiple-input multiple-output (MIMO) system has a great potential to enhance the throughput in the framework of wireless cellular networks [4], and

H.-L. Xiao $(\varangle) \cdot$ S. Ouyang

School of Information and Communication, Guilin University of Electronic Technology,

Guilin 541004, China

e-mail: xhl_xiaohailin@yahoo.com.cn

H.-L. Xiao - C.-X. Wang

Joint Research Institute for Signal and Image Processing, School of Engineering and Physical Sciences, Heriot-Watt University, Edinburgh EH14 4AS, UK 
the multiple-antenna Gaussian broadcast channels (BCs) have become the subject of intense research. This surge of interest has been spurred by the seminal work of Diaz and Simeone [5]. Recently, this region has been shown by Al-Naffouri et al. [6] to exhaust the sum-rate capacity of the channels, but the effect of spatial correlation on various multiuser scheduling schemes for MIMO-BCs has high complexity at the base station (BS). MIMO-BCs with power constraint whose capacity has been obtained [7], where a very large number of SUs is crucial to achieve a high channel capacity. However, the exhaustive search of selecting the best set of SUs is very complex. In this paper, we adopt a duality technique and dirty paper coding (DPC) to simplify the channels so that we can avoid the exhaustive search of selecting SUs and reduce complexity at the BS. In addition, a soft antenna trade-off scheme between different quality-of-service (QoS) groups in MIMO-BCs has been proposed, and the proposed scheme could improve QoS while simultaneously maximizing the total throughput (see [8] and references therein).

A new type of spectrum sharing transmission scheme has recently been proposed in [9], where multiple antennas are employed at the CR to enhance spectrum efficiency and throughput, and avoid causing detrimental interference to the PUs. We find an achievable region for cognitive MIMO Gaussian channels in a fashion analogous to [10], and the sum-rate capacity is a minimax problem in which the inner maximization corresponds to computing the capacity of a MIMO multiple access channel subject to a sum power constraint, but the algorithm of saddle point problems is very complicated [11]. Another notable works have investigated a different CR-MIMO channels, under this setting, the CR opportunistically uses primary spectrum when the PUs are sensed to be absent in that band [12,13]. However, control and coordination between the cognitive transmitter receiver pair are required to ensure the use of same spectrum.

Notation we use boldface letters to denote vectors and matrices. $\operatorname{Tr}(\cdot), \operatorname{det}(\cdot)$ and $\mathrm{E}[\cdot]$ denote the trace, determinant and expectation of a matrix, respectively. For any general matrix $\boldsymbol{H}, \boldsymbol{H}^{+}$denotes the conjugate transpose, $\boldsymbol{H}^{H}$ denotes matrix $\boldsymbol{H}$ transpose. $\boldsymbol{I}_{n}$ is the $n \times n$ identity matrix and $\boldsymbol{H} \geq 0$ denotes a positive semidefinite matrix. $\boldsymbol{X}^{n}$ denotes the row vector $(x(1), x(2), \ldots, x(n))$.

\section{System Model}

We consider a CR-MIMO Gaussian broadcast channels as depicted in Fig. 1. Let $n_{p, r}$ and $n_{c, r}$ denote the number of receiver antennas for the PUs and SUs, respectively. "Joint source" transmitter has $n_{t}$ multiple antennas. The transmit receiver signal is from "joint source" transmitters to the PUs and SUs, respectively.

Fig. 1 CR-MIMO Gaussian

broadcast channels model

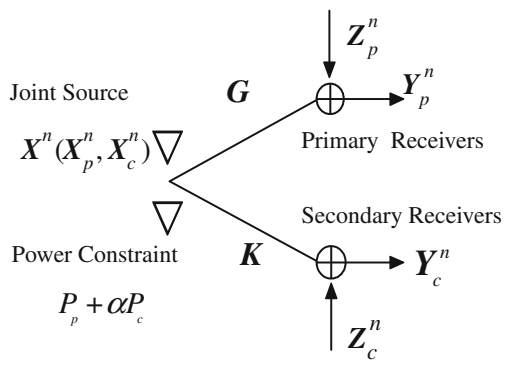


Let $\boldsymbol{X}_{p}^{n}$ and $\boldsymbol{X}_{c}^{n}$ be the vector signal transmitted by "Joint source". Let $\boldsymbol{G}$ and $\boldsymbol{K}$ be constant channel gain matrices as shown in Fig. 1, respectively. The additive noise at the primary and secondary receivers are Gaussian, independent and represented by $\boldsymbol{Z}_{p}^{n}$ and $\boldsymbol{Z}_{c}^{n}$, respectively, where $\boldsymbol{Z}_{p}^{n} \sim \boldsymbol{N}\left(0, \boldsymbol{I}_{n_{p, r}}\right)$ and $\boldsymbol{Z}_{c}^{n} \sim \boldsymbol{N}\left(0, \boldsymbol{I}_{n_{c, r}}\right) . \boldsymbol{Z}_{p}^{n}$ and $\boldsymbol{Z}_{c}^{n}$ can be arbitrary correlated between themselves, but this correlation does not impact the sum-rate capacity of the system as the primary and secondary decoders do not cooperate with each other [14]. The received vectors of the PUs and SUs are denoted by $\boldsymbol{Y}_{p}^{n}$ and $\boldsymbol{Y}_{c}^{n}$, respectively.

With the above model and notations, we can respectively describe the system at the $i$ th primary and secondary receivers by

$$
\begin{aligned}
\boldsymbol{Y}_{p}(i) & =\boldsymbol{G} \boldsymbol{X}_{p}(i)+\boldsymbol{Z}_{p}(i) \\
\boldsymbol{Y}_{c}(i) & =\boldsymbol{K} \boldsymbol{X}_{c}(i)+\boldsymbol{Z}_{c}(i)
\end{aligned}
$$

In the non-CR setting, a duality technique is used to transform MIMO-BCs into MIMO multiple access channels (MACs), which can be solved in an efficiency manner. In CR setting, the problem can also transform, but the transformation has many constraint conditions, including a sum power constraint and an interference constraint. Those constraints can be mathematically formulated as follows

$$
\begin{gathered}
q_{p}\left(\operatorname{Tr}\left(\Sigma_{p}^{n}\right)-P_{p}\right)+q_{c}\left(\operatorname{Tr}\left(\Sigma_{c}^{n}\right)-P_{c}\right) \leq 0 \\
\Sigma_{p}^{n} \geq 0, \quad \operatorname{Tr}\left(\Sigma_{p}^{n}\right) \leq P_{p}, \quad \Sigma_{c}^{n} \geq 0, \quad \operatorname{Tr}\left(\Sigma_{c}^{n}\right) \leq P_{c} \\
\operatorname{Tr}\left(\Sigma_{p}^{n}\right)+\operatorname{Tr}\left(\Sigma_{c}^{n}\right) \leq P_{p}+\alpha P_{c}
\end{gathered}
$$

where $q_{p}$ and $q_{c}$ are the auxiliary variables related to the respective interference constraint and sum power constraint. The covariance matrices of the primary and secondary input signal are $\Sigma_{p}^{n}$ and $\Sigma_{c}^{n}$, respectively, the traces of $\Sigma_{p}^{n}$ and $\Sigma_{c}^{n}$ satisfies the sum power constraint $P_{p}+\alpha P_{c}$. This new problem is concave in the covariance matrices $\boldsymbol{\Sigma}_{p}^{n}$ and $\boldsymbol{\Sigma}_{c}^{n}$ and convex in the scalar $\alpha(\alpha>0)$ with linear power constraints and is thus a convex-concave game, which can be solved by using interior point methods or other equivalent convex optimization methods. Once the optimal $\Sigma_{p}^{n}$ and $\Sigma_{c}^{n}$ are obtained, we can apply the BCs-to-MACs transformation to obtain the optimal transmit policies. So we use duality technique [15], the channels of the Fig. 1 can be also illustrated in Fig. 2.

Where multiple antennas $n_{t}$ of the "joint source" transmitters divide two parts. Let $n_{p, t}$ and $n_{c, t}$ denote the antenna numbers of primary and secondary transmitters, respectively. Note that the channels gain matrices are respectively given by $\boldsymbol{H}_{p, p}, \boldsymbol{H}_{c, p}, \boldsymbol{H}_{c, c}$ and $\boldsymbol{H}_{p, c}$, and are assumed to be Gaussian channels. It is assumed that the primary receivers know

Fig. 2 CR-MIMO Gaussian broadcast channels model by using a duality technique

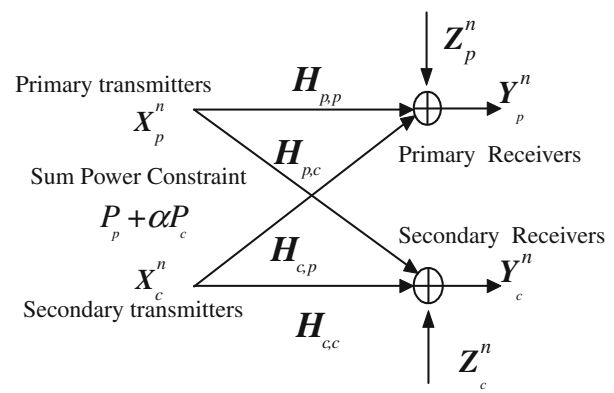


Fig. 3 Simplified CR-MIMO

Gaussian broadcast channels model by duality technique and dual DPC scheme

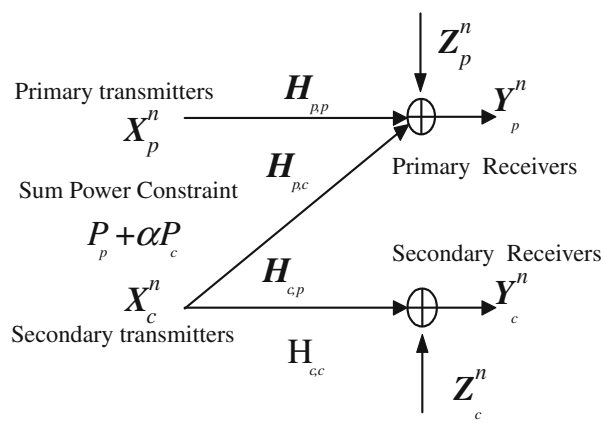

$\boldsymbol{H}_{p, p}, \boldsymbol{H}_{c, p}$, the primary transmitters know $\boldsymbol{H}_{p, p}$. It is also assumed that the secondary transmitters know $\boldsymbol{H}_{c, p}, \boldsymbol{H}_{c, c}, \boldsymbol{H}_{p, c}$ and the secondary receivers know $\boldsymbol{H}_{c, c}, \boldsymbol{H}_{p, c}$. The $i$ th primary and secondary receivers are respectively represented as

$$
\begin{aligned}
& \boldsymbol{Y}_{p}(i)=\boldsymbol{H}_{p, p} \boldsymbol{X}_{p}(i)+\boldsymbol{H}_{c, p} \boldsymbol{X}_{c}(i)+\boldsymbol{Z}_{p}(i) \\
& \boldsymbol{Y}_{c}(i)=\boldsymbol{H}_{p, c} \boldsymbol{X}_{p}(i)+\boldsymbol{H}_{c, c} \boldsymbol{X}_{c}(i)+\boldsymbol{Z}_{c}(i)
\end{aligned}
$$

In [6], the authors extended the capacity theorem by verifying that the sum-rate capacity of the Gaussian MIMO-BCs is precisely the DPC rate region. The capacity of dual DPC scheme has been obtained in [16]. The capacity of the CR-MIMO Gaussian broadcast channels can therefore be expressed as

$$
\begin{gathered}
\mathrm{C}_{p} \leq \log \operatorname{det}\left(\boldsymbol{I}_{n}+\boldsymbol{G}_{p} \boldsymbol{\Sigma}_{p}^{n} \boldsymbol{G}_{p}^{+}+\boldsymbol{H}_{c, p} \boldsymbol{\Sigma}_{c, c}^{n} \boldsymbol{H}_{c, p}^{+}\right)-\log \operatorname{det}\left(\boldsymbol{I}_{n}+\boldsymbol{H}_{c, p} \boldsymbol{\Sigma}_{c, c}^{n} \boldsymbol{H}_{c, p}^{+}\right) \\
\mathrm{C}_{\mathrm{c}} \leq \log \operatorname{det}\left(\boldsymbol{I}_{n}+\boldsymbol{H}_{c, c} \boldsymbol{\Sigma}_{c, c}^{n} \boldsymbol{H}_{c, c}^{+}\right)
\end{gathered}
$$

where the codeword $\boldsymbol{X}_{c, c}(i)$ is generated using Costa precoding by treating $\boldsymbol{H}_{p, p} \boldsymbol{X}_{p}(i)+$ $\boldsymbol{H}_{c, c} \boldsymbol{X}_{c, p}(i)$ as noncausally known interference. A characteristic feature of Costa's precoding is that $\boldsymbol{X}_{c, c}(i)$ is independent of $\boldsymbol{X}_{c, p}(i)$, and $\boldsymbol{X}_{c, c}(i)$ is distributed as $\boldsymbol{X}_{c, c}(i) \sim \boldsymbol{N}\left(0, \boldsymbol{\Sigma}_{c, c}^{n}\right)$. The two codewords $\boldsymbol{X}_{c, p}(i)$ and $\boldsymbol{X}_{c, c}(i)$ are superimposed to form the cognitive codeword $\boldsymbol{X}_{c}(i)=\boldsymbol{X}_{c, p}(i)+\boldsymbol{X}_{c, c}(i)$.

In CR-MIMO Gaussian channels, the primary receivers receive $\boldsymbol{H}_{p, p} \boldsymbol{X}_{p}(i)+\boldsymbol{H}_{c, p}$ $\left(\boldsymbol{X}_{c, p}(i)+\boldsymbol{X}_{c, c}(i)\right)+\boldsymbol{Z}_{p}(i)$. They treat $\boldsymbol{H}_{p, p} \boldsymbol{X}_{p}(i)+\boldsymbol{H}_{c, p} \boldsymbol{X}_{c, p}(i)$ as the valid codeword and $\boldsymbol{H}_{c, p} \boldsymbol{X}_{c, c}(i)+\boldsymbol{Z}_{p}(i)$ as Gaussian noise. The secondary receivers receive $\boldsymbol{H}_{p, c} \boldsymbol{X}_{p}(i)+$ $\boldsymbol{H}_{c, c}\left(\boldsymbol{X}_{c, p}(i)+\boldsymbol{X}_{c, c}(i)\right)+\boldsymbol{Z}_{c}(i)$. Taking $\boldsymbol{G}_{p}=\left[\boldsymbol{H}_{p, p} \boldsymbol{H}_{c, p}\right]$ and $\boldsymbol{\Sigma}_{p}^{n}=\left[\begin{array}{cc}\boldsymbol{\Sigma}_{p}^{n} & \boldsymbol{Q}^{n} \\ \boldsymbol{Q}^{n+} & \boldsymbol{\Sigma}_{c, p}^{n}\end{array}\right]$, where $\boldsymbol{Q}^{n}=\mathrm{E}\left[\boldsymbol{X}_{p}^{n},\left(\boldsymbol{X}_{c, p}^{n}\right)^{+}\right]$. The noncausally known interference $\boldsymbol{H}_{p, c} \boldsymbol{X}_{p}(i)+\boldsymbol{H}_{c, c} \boldsymbol{X}_{c, p}(i)$ is cancelled by the Costa precoder in dual DPC scheme [16]. So the channels as shown as Fig. 2 can be simplified as Fig. 3

The received signal vectors at the $i$ th primary and secondary receivers, $\boldsymbol{Y}_{p}(i)$ and $\boldsymbol{Y}_{c}(i)$, are given, respectively, as

$$
\begin{gathered}
\boldsymbol{Y}_{p}(i)=\boldsymbol{H}_{p, p} \boldsymbol{X}_{p}(i)+\boldsymbol{H}_{c, p} \boldsymbol{X}_{c}(i)+\boldsymbol{W}_{p}(i) \\
\boldsymbol{Y}_{c}(i)=\boldsymbol{H}_{c, c} \boldsymbol{X}_{c}(i)+\boldsymbol{W}_{c}(i)
\end{gathered}
$$

where the noise vectors $\boldsymbol{W}_{p}(i)$ and $\boldsymbol{W}_{c}(i)$ are Gaussian and are also assumed to be independent identically distributed (i.i.d). Finally, the channel capacity of the PUs after whitening the interference [17] is given by 


$$
\mathrm{C}_{p}=\log \operatorname{det}\left(\boldsymbol{I}_{n}+\frac{\left(P_{p}+\alpha P_{c}\right)}{N} \boldsymbol{G}_{p} \boldsymbol{G}_{p}^{H}\left(\boldsymbol{\Sigma}_{p}^{n}\right)^{-1}\right)
$$

Note that the interference vector is a scalar multiplied Gaussian signal vector with $N$ independent data streams. The interference signal vector and the noise signal vector have the same statistical characteristics so that the two terms can be represented as a single random vector. The channel capacity for the SUs is given by

$$
\mathrm{C}_{c}=\log \operatorname{det}\left(\boldsymbol{I}_{n}+\frac{\alpha P_{c}}{N N_{0}} \boldsymbol{H}_{c, c}\left(\boldsymbol{H}_{c, p}^{H} \boldsymbol{H}_{c, p}\right)^{-1} \boldsymbol{H}_{c, c}^{H}\right)
$$

where $N_{0}$ denotes additive Gaussian noise power spectrum.

\section{Sum-Rate Capacity Analysis}

The sum-rate capacity of the CR-MIMO Gaussian broadcast channels can be represented as

$$
\mathrm{C}=\operatorname{argmax}\left\{\mu \mathrm{C}_{p}+\mathrm{C}_{c}+\sum_{i}^{n} \lambda_{i}\left(\operatorname{Tr}\left(\Sigma_{p}^{n}\right)+\operatorname{Tr}\left(\Sigma_{c}^{n}\right)-P_{p}-\alpha P_{c}\right)\right\}
$$

where $\lambda_{i}$ is Lagrange multipliers. Such that for any scalar $\mu>0, \arg \max \left(\mu \mathrm{C}_{p}+\mathrm{C}_{c}\right)$ is a point on the boundary of the sum-rate capacity of the CR-MIMO Gaussian broadcast channels if the condition given by (5) is satisfied for $\alpha$. According to Kuhn-Tucker theorem [18,19], we can obtain

$$
\nabla\left(\mu \mathrm{C}_{p}+\mathrm{C}_{c}\right)+\sum_{i}^{n} \lambda_{i} \nabla\left[\operatorname{Tr}\left(\Sigma_{p}^{n}\right)+\operatorname{Tr}\left(\Sigma_{c}^{n}\right)-P_{p}-\alpha P_{c}\right]=0
$$

We define Lagrange function $\varphi(C)$ as

$$
\begin{gathered}
\varphi(C)=f(x)+\sum_{i}^{n} \lambda_{i} g(C) \\
f(C)=\mu \mathrm{C}_{p}+\mathrm{C}_{c} \\
g(C)=\operatorname{Tr}\left(\Sigma_{p}^{n}\right)+\operatorname{Tr}\left(\Sigma_{c}^{n}\right)-P_{p}-\alpha P_{c}
\end{gathered}
$$

When the power constraints are satisfied with inequality (5), then $\operatorname{Tr}\left(\Sigma_{p}^{n}\right)+\operatorname{Tr}\left(\Sigma_{c}^{n}\right)-P_{p}-\alpha P_{c}$ is negative. However, (15) is equality constraint. Hence, for any $\lambda_{i}$ will take the value zero. When the power constraint is satisfied with equality, then

$$
\operatorname{Tr}\left(\Sigma_{p}^{n}\right)+\operatorname{Tr}\left(\Sigma_{c}^{n}\right)-P_{p}-\alpha P_{c}=0
$$

The complementary slackness condition is satisfied. We find an efficiency solvable dual problem for the (3) by using an iterative power allocation algorithm. In [19], the authors have proposed the sub-gradient iterative power allocation (SIPA) algorithm to solve sumrate optimization problem for CR-MIMO broadcast channels, where the algorithm needs to determine the optimal $\lambda$ (where $\lambda$ can be viewed as a water-level in the water-filling principle). However, the Lagrangian function $g(\lambda)$ is convex over $\lambda$, the optimal $\lambda$ can be obtained via the one-dimensional search. To reduce its complexity, we use zero-forcing beamforming (ZFBF) technique. The basic idea of ZFBF is that each user is assigned one column vector of the pseudoinverse of the downlink channel matrix [20]. Moreover, ZFBF can effectively 
eliminate interuser interference and improve channel capacity. In this paper, to maximize the sum-rate capacity using the ZFBF, the selected singular vectors must be nearly orthogonal to each other and their corresponding singular values be sufficiently large. In particular, by applying the singular value decomposition (SVD) to the channel matrices of all the users, we will only consider the singular vectors corresponding to maximum singular values.

We search the optimal $q_{p}, q_{c}$ through the proposed algorithm; this is, in each iterative step, we update the vector $\left[q_{p}, q_{c}\right]$. The steps of the algorithm are given in the following algorithm.

1. Initialization: $q_{p}^{(1)}=0.005, q_{c}^{(1)}=0.005, n=1$

2. Repeat $2 \mathrm{a}$

2a. Find the solution of the (14) through matrix $\boldsymbol{H}$ SVD and ZFBF algorithm.

2b. Update $q_{p}^{(1)}, q_{p}^{(n)}$ through the algorithm $q_{p}^{(n+1)}=q_{p}^{(n)}+t\left(\operatorname{Tr}\left(\Sigma_{p}^{n}\right)-P_{p}\right), q_{c}^{(n+1)}=$ $q_{c}^{(n)}+t\left(\operatorname{Tr}\left(\Sigma_{c}^{n}\right)-P_{c}\right)$,

2c. $n=n+1$

3. Stop when $\left|q_{p}^{(n)}\left(\operatorname{Tr}\left(\boldsymbol{\Sigma}_{p}^{n}\right)-P_{p}\right)\right| \leq \varepsilon$ and $\left|q_{c}^{(n)}\left(\operatorname{Tr}\left(\boldsymbol{\Sigma}_{c}^{n}\right)-P_{c}\right)\right| \leq \varepsilon^{+}$are satisfied simultaneously.

where $t$ denotes the step size of the algorithm, $\varepsilon, \varepsilon^{+}$are infinitely small quantity $(\varepsilon>0)$.

The above proposed algorithm uses to prove the convergence of (15) and also allows us to characterize sum-rate capacity of the (17). A point $C^{*}=\left(C_{p}^{*}, C_{c}^{*}\right)$ is on the boundary of the sum-rate capacity if and only if there exists a $\mu \geq 0$ such that the linear function $\mu \mathrm{C}_{p}+\mathrm{C}_{c}$ achieves its maximum, over all $\left(C_{p}, C_{c}\right)$ in the region, at $C^{*}$. Suppose that there exist two points $C^{(1)}=\left(C_{p}^{(1)}, C_{c}^{(1)}\right)$ and $C^{(2)}=\left(C_{p}^{(2)}, C_{c}^{(2)}\right)$ that are on the boundary of this region. Let be corresponding parameters in (8) and (9) for which the inequalities become tight. Then, for any $\lambda \in[0,1]$, we have that

$$
\begin{aligned}
\lambda C_{c}^{(1)}+(1-\lambda) C_{c}^{(2)} \leq & \log \operatorname{det}\left(\boldsymbol{I}_{n}+\boldsymbol{H}_{c, c} \boldsymbol{\Sigma}_{c^{(1)}, c^{(1)}}^{n} \boldsymbol{H}_{c, c}^{+}\right) \\
& +(1-\lambda) \log \operatorname{det}\left(\boldsymbol{I}_{n}+\boldsymbol{H}_{c, c} \boldsymbol{\Sigma}_{c^{(2)}, c^{(2)}}^{n} \boldsymbol{H}_{c, c}^{+}\right) \\
\leq & \log \operatorname{det}\left(\boldsymbol{I}_{n}+\boldsymbol{H}_{c, c} \boldsymbol{\Sigma}_{c^{*}, c^{*}}^{n} \boldsymbol{H}_{c, c}^{+}\right)
\end{aligned}
$$

where $C^{*} \stackrel{\text { def }}{=} \lambda C_{c}^{(1)}+(1-\lambda) C_{c}^{(2)}$ and the last inequality follows from Jensen's inequality. Similarly

$$
\begin{aligned}
& \lambda\left(C_{p}^{(1)}+C_{c}^{(1)}\right)+(1-\lambda)\left(C_{p}^{(2)}+C_{c}^{(2)}\right) \\
& \quad \leq \log \operatorname{det}\left(\frac{\boldsymbol{I}_{n}+G_{p} \boldsymbol{\Sigma}_{p^{*}}^{n} \boldsymbol{G}_{p}^{+}+H_{c, p} \boldsymbol{\Sigma}_{c^{*}, c^{*}}^{n} \boldsymbol{H}_{c, p}^{+}}{\left(\boldsymbol{I}_{n}+\boldsymbol{H}_{c, p} \boldsymbol{\Sigma}_{c^{*}, c^{*}}^{n} \boldsymbol{H}_{c, p}^{+}\right)\left(\boldsymbol{I}_{n}+\boldsymbol{H}_{c, c} \boldsymbol{\Sigma}_{c^{*}, c^{*}}^{n} \boldsymbol{H}_{c, c}^{+}\right)}\right)
\end{aligned}
$$

As discussed above, there exists a $\mu \geq 0$ such that the linear function $\mu \mathrm{C}_{p}+\mathrm{C}_{c}$ achieves its maximum. After whitening the interference is adopted, the maximum channels capacity of simplified CR-MIMO Gaussian broadcast channels are uniquely obtained as (17).

\section{Numerical Simulation Results}

In this section, numerical simulation results are provided to examine the performance of our proposed algorithm with finite numbers of users and antennas. Moreover, we also 


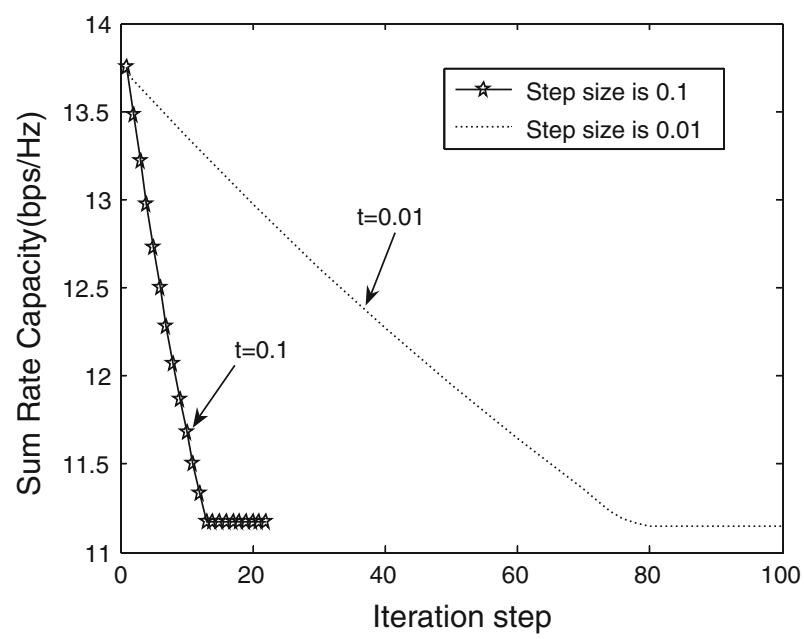

Fig. 4 The convergence behaviour of the proposed algorithm, parameter $t$ denotes the step size of the algorithm

provide some numerical simulation results on the sum-rate capacity of the CR-MIMO Gaussian broadcast channels. In CR-MIMO Gaussian broadcast channels, the channel coefficients are real and also restrict ourselves to real inputs and outputs. The noise covariance matrix is assumed to be the identity matrix. Ten thousand channel matrices are generated with Monte Carlo simulations. We consider the two primary and secondary transmitters have two antennas each, and the two primary and secondary receivers have two antennas, respectively. In addition, power constraint parameters are satisfied $P_{p}=10, P_{c}=10 \mathrm{~dB}$, respectively. Following parameter values are used in all numerical simulations unless stated otherwise: $\varepsilon$ and $\varepsilon^{+}$are small-valued positive numbers and equal to 0.005 , respectively.

Figure 4 plots the sum-rate capacity versus the number of iterations of the proposed iterative power allocation algorithm for step sizes $t=0.1$ and $t=0.01$. As can be seen from the figure, the step size affects the accuracy and convergence speed of the algorithm. Numerical simulation results for the maximum sum-rate capacity are also presented in Fig. 4 to examine the performance of our proposed algorithm in practical networks with a finite number of users.

In Fig. 5, It is show that the channel capacity $\mathrm{C}_{p}$ decreases with increasing the value of scalar $\alpha$, this will corresponding to using the sum power to support the PUs. The proposed channel outperformes PUs' capacity at the cost of the SUs' capacity.

\section{Conclusions}

The CR-MIMO Gaussian broadcast channels have been studied where multiple antennas are available for both PUs and SUs in a spectrum sharing environment, and the sum-rate capacity has been obtained under both the SUs' transmit power constraint and interference power constraint at the primary receivers. In this paper, we have firstly adopted a duality technique and dirty paper DPC to simplify the channels. And then, we have proposed an iterative power allocation algorithm to obtain the optimal sum-rate capacity and examined the effects of the constraint parameters on the concerned quantities. Finally, the numerical simulation results showed that the step size affected the accuracy and convergence speed of the algorithm, and the proposed channel outperformed PUs' capacity at the cost of the SUs' capacity. 


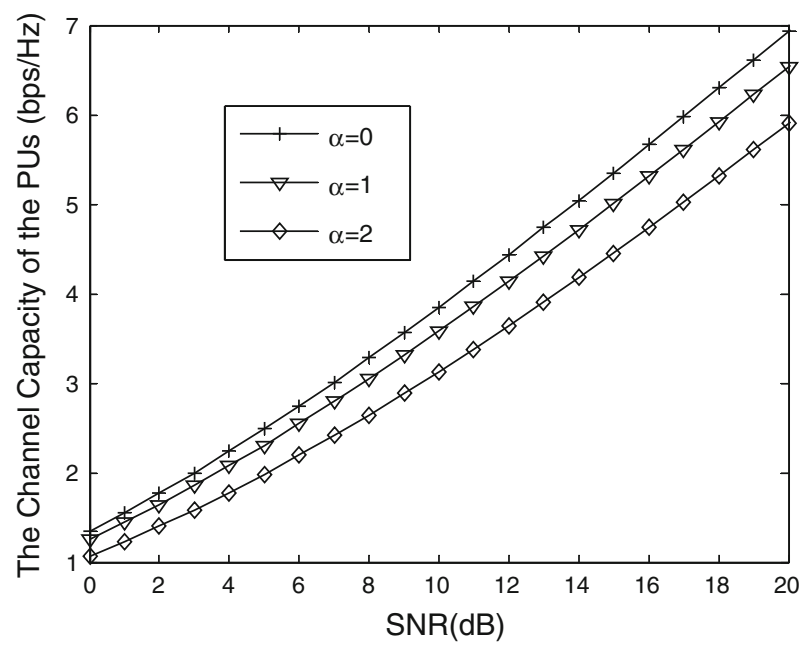

Fig. 5 The channel capacity of the PUs for varying $\alpha$

Acknowledgements The work of H.-L. Xiao and S. Ouyang is supported by the National Basic Research Program of China "973" (Grant No.: 2008CB317109), Guangxi Natural Science Foundation (No.: 2011GXNSFD018028 and 0991241), NSFC (Grant No. 60972084). H.-L. Xiao and C.-X. Wang acknowledge the support from the Scottish Funding Council for the Joint Research Institute in Signal and Image Processing with the University of Edinburgh, as part of the Edinburgh Research Partnership in Engineering and Mathematics (ERPem), and the support from the RCUK for the UK-China Science Bridges: R \& D on (B)4G Wireless Mobile Communications.

\section{References}

1. Hong, X., Chen, Z., Wang, C. X., Vorobroy, S., \& Thompson, J. S. (2009). Cognitive radio networks: Interference cancellation and management techniques. IEEE Vehicular Technology Magazine, 4, 76-84.

2. Choi, K. W. (2010). Adaptive sensing technique to maximize spectrum utilization in cognitive radio. IEEE Transactions on Vehicular Technology, 59, 992-998.

3. Wang, C. X., Hong, X., Ge, X., Cheng, X., Zhang, G., \& Thompson, J. S. (2010). Cooperative MIMO channel models: A survey. IEEE Communications Magazine, 48, 80-87.

4. Xiao, H. -L., Ouyang, S., \& Nie, Z. P. (2010). Design and performance analysis of compact planar inverted-L diversity antenna for handheld terminals. Wireless Personal Communications, 52, 709-718.

5. Diaz, J., Simeone, O., \& Bar-Ness, Y. (2008). Asymptotic analysis of reduced-feedback strategies for MIMO gaussian broadcast channels. IEEE Transactions on Information Theory, 54, 1308-1316.

6. Al-Naffouri, T. Y., Sharif, M., \& Hassibi, B. (2009). How much does transmit correlation affect the sum-rate scaling of MIMO Gaussian broadcast channels?. IEEE Transactions on Communications, 56, 562-572.

7. Wang, C., Hong, X., Chen, H. H., \& Thompson, J. (2009). On capacity of cognitive radio networks with average interference power constraints. IEEE Transactions on Wireless Communications, 8, 1620-1625.

8. Lee, S. H., \& Thompson, J. (2010). Trade-off multiplexing streams in MIMO broadcast channels. IEEE Communications Letters, 14, 115-117.

9. Gao, F., Zhang, R., Liang, Y. C., \& Wang, X. D. (2010). Design of learning based MIMO cognitive radio systems. IEEE Transactions on Vehicular Technology, 59, 1707-1720.

10. Yin, H., \& Liu, H. (2002). Performance of space-division multiple-access with scheduling. IEEE Transactions on Wireless Communications, 1, 611-618.

11. Zhang, R., \& Liang, Y. C. (2008). Exploiting multi-antennas for opportunistic spectrum sharing in cognitive radio networks. IEEE Journal of Selected Topics in Signal Processing, 2, 88-102.

12. Scutari, G., \& Palomar, D. P. (2010). MIMO cognitive radio: A game theoretical approach. IEEE Transactions on Signal Processing, 58, 761-780. 
13. Gomadam, K. S., \& Jafar, S. A. (2010). Duality of MIMO multiple access channel and broadcast channel with amplify and forward relays. IEEE Transactions on Communications, 58, 211-217.

14. Soundararajan, R., \& Vishwanath, S. (2008). Adaptive sum power iterative waterfilling for MIMO cognitive radio channels. IEEE international conference on communications (pp. 1060-1064). Beijing, China.

15. Jafar, S. A., \& Shamai, S. (2008). Degrees of freedom region of the MIMO X channel. IEEE Transactions on Information Theory, 54, 151-169.

16. Peng, Y., \& Rajan, D. (2010). Capacity bounds for a cognitive MIMO Gaussian Z-interference channel. IEEE Transactions on Vehicular Technology, 59, 1865-1876.

17. Kang, M. S., Jung, B. C., \& Sung, D. K. (2008). A pre-whitening scheme in a MIMO-based spectrum-sharing environment. IEEE Communications Letters, 12, 831-833.

18. Liang, X. B. (2008). An algebraic, analytic, and algorithmic investigation on the capacity and capacityachieving input probability distributions of finite-input-finite-output discrete memoryless channels. IEEE Transactions on Information Theory, 54, 1003-1023.

19. Zhang, L., Xin, Y., \& Liang, Y. C. (2009). Weighted sum rate optimization for cognitive radio MIMO broadcast channels. IEEE Transactions on Wireless Communications, 8, 2950-2959.

20. Lu, P., \& Yang, H. C. (2010). Sum-rate analysis of multiuser MIMO system with zero-forcing transmit beamforming. IEEE Transactions on Communications, 57, 2585-2589.

\section{Author Biographies}
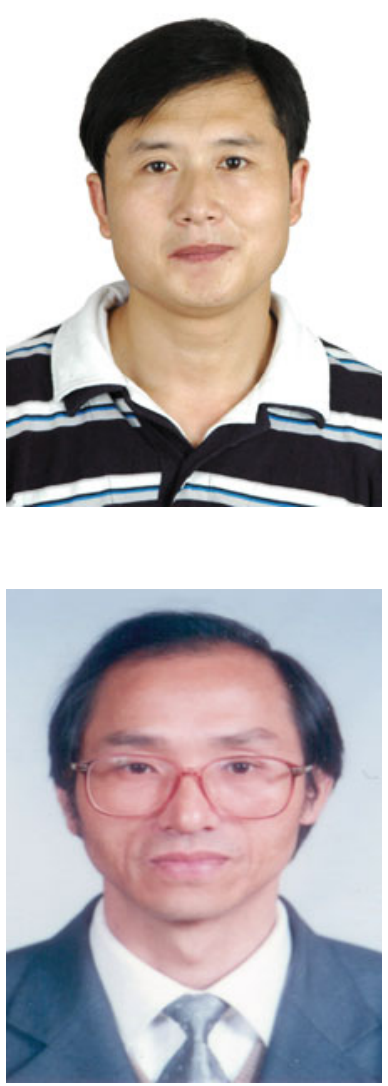

Hai-Lin Xiao was born in 1976. He received the B.S. and M.S. degrees from Wuhan University and Guangxi Normal University in 1998 and 2004 respectively. He received Ph.D. degree from the University of Electronic Science and Technology of China (UESTC) in 2007. He was Professor in the School of Information and Communications, Guilin University of Electronic Technology. Now he is a Research Fellow with Joint Research Institute for Signal and Image Processing, School of Engineering \& Physical Sciences, Heriot-Watt University. His research interests include MIMO wireless communications, cooperative communications and smart antenna techniques.

Shan Ouyang was born in 1960. He received the B.S. degree in electronic engineering from Guilin University of Electronic Technology, Guilin, China, in 1986 and the M.S. and Ph.D. degrees in electronic engineering from Xidian University, Xi' an, China, in 1992 and 2000, respectively. In 1986, he jointed Guilin University of Electronic Technology, where he is presently a Professor and the Dean of the School of Information and Communications. From June 2001 to May 2002, he was a Research Associate with the Department of Electronic Engineering, the Chinese University of Hong Kong. From January 2003 to January 2004, he was a Research Fellow with Department of Electronic Engineering, University of California at Riverside. His research interests are mainly in the areas of signal processing for communications and radar, adaptive filtering, and neural network learning theory and applications. Dr. Ouyang received the Outstanding Youth Award of the Ministry of Electronic Industry and Guangxi Province Outstanding Teacher Award, China, in 1995 and 1997, respectively. He received the National Excellent Doctoral Dissertation of China in 2002. 


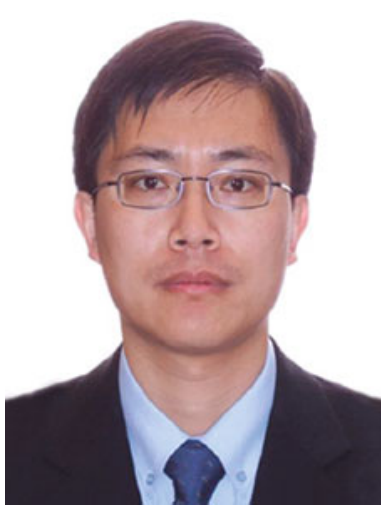

Cheng-Xiang Wang was born in 1975. He received the B.Sc. and M.Eng. degrees in Communication and Information Systems from Shandong University, China, in 1997 and 2000, respectively, and the Ph.D. degree in Wireless Communications from Aalborg University, Denmark, in 2004. He has been with Heriot-Watt University, Edinburgh, UK since 2005, first as a Lecturer and then as a Reader in 2009. $\mathrm{He}$ is also an Honorary Fellow of the University of Edinburgh, UK. He was a Research Fellow at the University of Agder, Grimstad, Norway, from 2001-2005, a Visiting Researcher at Siemens AG-Mobile Phones, Munich, Germany, in 2004. His research interests include wireless channel modelling and simulation, CR networks, vehicular communication networks, green radio communications, cooperative (relay) communications, cross-layer design of wireless networks, MIMO, OFDM, UWB, and (beyond) 4G wireless communications. 